\title{
ICONO14
}

ICONO 14, Revista de comunicación y tecnologías emergentes, 20(1), 2022. ISSN: 1697-8293

https://doi.org/10.7195/ri14.v20i1.1700

\section{La creatividad vernácula en la era digital: enseñanza de la comunicación publicitaria en entornos conectivos}

Vernacular Creativity in the Digital Age: Teaching Advertising Communication in Connective Environments

\section{Criatividade vernácula na era digital: O ensino da comunicação publicitária nos contextos conectivos}

Carolina Fernández-Castrillo ${ }^{1}$ (i)

Carla Rogel ${ }^{2}$ (D)

1 Profesora Ayudante Doctora (Universidad Carlos III de Madrid), España

${ }^{2}$ Jefa de marketing (Spain Media), España

Recibido: 30/12/2020; Revisado: 05/01/2021; Aceptado: 19/02/2021; Publicado: 01/01/2022

Para citar este artículo: Fernández-Castrillo, C. y Rogel, C. (2021). La creatividad vernácula en la era digital: enseñanza de la comunicación publicitaria en entornos conectivos, Icono 14, 20(1). doi: https://doi.org/10.7195/ri14.v20 $\underline{11.1700}$

\section{Resumen}

Esta investigación surge en respuesta a la demanda por parte del alumnado centennial y millennial de nuevas fórmulas para el desarrollo de la creatividad a través del uso de las nuevas tecnologías. El artículo plantea la necesidad de incorporar el apropiacionismo como estrategia 
educativa para promover la competencia creativa en la era digital. El objetivo principal del trabajo consiste en fomentar nuevas fórmulas didácticas que consientan la integración de las dinámicas sociotecnológicas que marcan las interacciones del día a día en la red. Se ha adoptado el mash-up como herramienta principal para llevar a cabo una propuesta metodológica de innovación docente en los estudios de comunicación publicitaria dentro del contexto académico. La construcción del modelo denominado lateral visual thinking supone una revisión del pensamiento de diseño en base a dos características distintivas de los procesos comunicativos en la actualidad: la creatividad vernácula y la práctica del pensamiento lateral mediante la recombinación de materiales de diverso origen y autoría en la World Wide Web.

Palabras clave: Apropiación; contenidos generados por usuarios; creatividad vernácula; foundfootage. mash-up; pensamiento lateral

\begin{abstract}
This research comes about in response to the demand of centennial and millennial students for new methods to develop creativity through use of new technologies. This paper considers the need to incorporate appropriation as an educational strategy to promote the creative competence in the digital age. The main goal of this work is to promote new didactic methods that allow the integration of social and technological dynamics that determine online daily interactions. The mashup has been adopted as the main tool to carry out a methodological proposal of teaching innovation in the academic context of Advertising Communication studies. The construction of the model called lateral visual thinking constitutes a revision of the design thinking based on two distinctive characteristics: vernacular creativity and the practice of lateral thinking through the recombination of materials of diverse origin and authorship in the World Wide Web.
\end{abstract}

Keywords: appropriation; found footage; lateral thinking; mash-up; user generated content; vernacular creativity

\title{
Resumo
}

Esta pesquisa surge em resposta à procura dos alunos centennials . milllenials, de disporem de novas fórmulas para o desenvolvimento da criatividade através do uso das novas tecnologias. $O$ artigo levanta a necessidade de integrar o apropriacionismo como estratégia educacional para promover a concorrência criativa na era digital. O objetivo principal do trabalho consiste em promover novas fórmulas didáticas que permitam a integração das dinâmicas sócio tecnológicas que, por sua vez, marcam as interações do dia-a-dia na Internet. O mash-up foi adotado como a principal ferramenta para a realização de uma proposta metodológica de inovação para o ensino nos estudos de comunicação publicitária no âmbito académico. A construção do modelo chamado lateral visual thinking envolve uma revisão do design thinking a partir de duas características distintas dos processos de comunicação de hoje: a criatividade vernacular e a prática do pensamento lateral, por meio da recombinação de materiais de diferentes origens e autorias na World Wide Web.

Palavras-chave: apropriação; found footage; lateral thinking; mash-up; user generated content; vernacular creativity 


\section{Introducción}

La promoción y protección de la creatividad es uno de los compromisos fundamentales adquiridos por las Naciones Unidas y sus estados miembros para garantizar la máxima libertad de expresión en la sociedad actual (UNESCO, 2018). En su último informe sobre los trabajos del futuro, el Foro Económico Mundial respalda esta necesidad situando a la creatividad en la tercera posición de las diez competencias principales para la Cuarta Revolución Industrial, frente al décimo puesto que ocupaba en la misma lista en el año 2015 (Gray, 2020). Por tanto, desde el sistema universitario urge fomentar el desarrollo del pensamiento creativo para formar a profesionales capaces de adelantarse al presente y de resolver nuevos desafíos a través de soluciones múltiples. Para entrenar este tipo de inteligencia, los sistemas educativos deberán adaptar su enfoque de modo que los estudiantes se sientan dueños de su propio proceso de aprendizaje, creando un fuerte sentido de comunidad a través del uso de las nuevas tecnologías de la información y del conocimiento (Foro Económico Mundial, 2020).

Según una reciente investigación realizada por Google, FAD y BBVA (Ballesteros et al., 2020), los jóvenes españoles consideran que el dominio tecnológico será la clave de su porvenir laboral. Por ello reclaman una mayor presencia de las tecnologías a nivel instrumental, estratégico y emocional a lo largo de su proceso educativo y una formación más sólida para la correcta gestión de la información disponible en la red, superando así el actual modelo de autocapacitación mediante el procedimiento de ensayo-error. Para alcanzar dicho objetivo, se requiere un cambio de perspectiva basado en métodos de instrucción más interactivos en los que los profesores adopten un rol de mediadores y abandonen el viejo modelo de enseñanza de corte jerárquico y unidireccional.

El presente estudio surge en respuesta a esta necesidad, pretendiendo brindar una nueva perspectiva resultante del influjo de los procesos colaborativos que han emergido mediante el uso de las redes sociales durante las dos primeras décadas del siglo XXI. En particular, nos centraremos en la enseñanza de la Publicidad, un sector en el que la presencia de los contenidos generados por usuarios ha revolucionado el modelo de interacción entre los distintos agentes participantes en el proceso de creación, producción y distribución de productos de corte creativo. En efecto, a partir del influjo de la Web 2.0, las marcas han comenzado a establecer un diálogo directo con los usuarios (branding), activando vínculos emocionales mediante la generación de contenidos de valor (branded content), mientras que los usuarios se han convertido en generadores de contenido asumiendo el viejo rol de las marcas (identidad digital como marca personal).

Con el fin de incorporar al ámbito educativo las dinámicas habituales en los entornos conectivos, nuestra propuesta metodológica se centrará en el apropiacionismo como marco conceptual de referencia. La reutilización y distribución de materiales existentes marca el día a día en las interacciones on-line y se ha convertido en la base de la mayoría de las prácticas mediáticas en la era del remix. La ausencia de este tipo de procesos en un 
sistema educativo excesivamente planificado impide "la interpelación, la interpretación y la interacción entre los agentes implicados en el acto educativo" (Agudelo, 2019, p. 11). Tal y como señala Agudelo, "las prácticas mediáticas ya no son definidas estrictamente como recepciones, sino como apropiaciones, entonces éstas pueden también ser entendidas como prácticas creativas, constructivas e interpretativas por parte de la audiencia" (2019, p. 2).

Pese a la aparente novedad de esta estrategia, se trata de un fecundo método creativo con una larga historia en el sector artístico. En este caso, nos consentirá integrar el contexto popular-mediático (Martín-Barbero, 1987) en las aulas para el desarrollo de un pensamiento crítico, lateral y colaborativo. Dentro de las distintas expresiones apropiacionistas, adoptaremos el mash-up como instrumento básico para la propuesta de una metodología de innovación docente en la que llevaremos a cabo una adaptación al ecosistema digital de las aportaciones de De Bono (1989) sobre el uso consciente del pensamiento lateral a modo de técnica para liberar la mente de los procesos de análisis y de razonamiento característicos del pensamiento lógico o vertical.

\section{Método}

Este artículo analiza la utilidad del apropiacionismo como recurso pedagógico dentro del Espacio Europeo de Educación Superior y, más concretamente, en la rama publicitaria. Se plantean los siguientes objetivos:

1)Responder a la demanda de las nuevas generaciones de una formación universitaria más innovadora, participativa y tecnológica.

2) Incorporar métodos de pensamiento lateral, design thinking y creación colectiva del conocimiento para el desarrollo de la competencia creativa.

3)Integrar nuevos géneros y formatos audiovisuales a la enseñanza publicitaria como incentivo para reflexionar en torno a las nuevas posibilidades creativas en la era digital.

Desde el punto de vista metodológico, estamos frente a un estudio mixto de tipo descriptivo-exploratorio. En primer lugar, se ha procedido a la revisión de las principales teorías sobre el desarrollo de la creatividad y, posteriormente, se ha llevado a cabo un examen crítico para analizar el impacto de estas en la era digital. Partiendo del estudio de los rasgos generacionales propios de millennials y centennials, y habiendo comprobado el lugar que ocupa el desarrollo creativo a través del uso de las nuevas tecnologías en el plan de estudio del Grado de Publicidad y Relaciones Públicas, se ha procedido a la propuesta de un método didáctico en respuesta a las posibles carencias actuales. Para la propuesta de esta nueva metodología, se han adoptado como puntos de referencia las teorías sobre pensamiento lateral, el método del design thinking y la técnica del found footage. El resultado final es la introducción de una nueva metodología docente denominada lateral visual thinking y el diseño de una actividad de carácter innovador. 


\section{La competencia creativa en la era del produsuario}

La aproximación científica a la creatividad es compleja, al responder a la interrelación de una amplia variedad de perspectivas que engloban la producción, el pensamiento, la personalidad y la inteligencia (Morales, Aguilar y Rodríguez, 2018). En la actualidad, la creatividad se mide en términos de destrezas para afrontar situaciones imprevistas, trabajar en equipo, proponer soluciones, obtener el mayor rendimiento posible de la tecnología y aplicar la lógica competitiva (Luengo, Luzón y Torres, 2008). Entre los principios a tener en cuenta para el desarrollo de la competencia creativa, se encuentran la "motivación, fluidez, iniciativa, adopción de riesgos y elaboración de respuestas alternativas" (Porto, 2008, p. 83).

Se considera una persona competente aquella capaz de combinar de forma adecuada conocimientos, capacidades y habilidades con sus recursos cognitivos, personales y sociales (Perrenoud, 2000). Así pues, quedan obsoletos los viejos planteamientos educativos centrados en la memorización y la repetición, al cederse el protagonismo a las habilidades cognitivas y fundamentalmente creativas (Porto, 2008). Se trata de un cambio estructural mediante el que se persigue motivar al alumno en lugar de únicamente evaluarle (Trillo, 2005). Por lo tanto, no es el profesor quien enseña, sino el alumno quien aprende (Domínguez, 2011). La aplicación de la competencia creativa requiere la reevaluación por parte del estudiante del concepto de originalidad basándose en su propia individualidad, así como la motivación por parte del personal docente a través de la atención a sus demandas y necesidades. De este modo, se persigue alcanzar una mayor fluidez en el ritmo de enseñanza, adaptándose a las condiciones individuales y promoviendo, por último, el sentido de la responsabilidad desde la libertad y el desarrollo de la imaginación.

En nuestros días, la práctica del remix se ha beneficiado de la democratización y accesibilidad a la creación de contenidos y está al alcance de todos los usuarios. Hemos venido observando que a menudo los millennials y centennials recurren a esta técnica de forma intuitiva con resultados altamente creativos. Consideramos que una correcta guía para los estudiantes mediante un método adecuado y las herramientas oportunas supondría un soporte excelente para entender el discurso publicitario en la era del produsuario (Bruns, 2008).

\subsection{El pensamiento lateral y el razonamiento abstracto}

De Bono y Gombau (2008) desarrollaron el concepto de "pensamiento lateral" para referirse a un enfoque alternativo y multiperspectivo para la resolución de problemas, desde una posición completamente alejada del pensamiento lógico y lineal. Se trata de uno de los métodos creativos imprescindibles por su planteamiento provocador y su acreditada eficacia. Insistimos en que la lateralidad hace referencia a un tipo de razonamiento creativo 
y perceptivo que permite analizar un conflicto desde perspectivas alternativas basadas en la originalidad, la inventiva y la flexibilidad, consiguiendo así una mayor libertad y fluidez de ideas (Villarroel, 2011). A diferencia del pensamiento lógico o vertical, éste se basa en la libertad, la irracionalidad, la flexibilidad y el ingenio en la asociación de conceptos, la elaboración de hipótesis y el método deductivo (Prado, Viteri y Rojas, 2017).

Ante nuevos desafíos y contextos de grandes cambios, se requiere el desarrollo del razonamiento abstracto, basado en la planificación, la elección de los objetivos y la representación mental de los conceptos. Asimismo, resulta imprescindible la conexión con la inteligencia emocional, en términos de personalidad, autoestima y estados de ánimo, para refinar la capacidad de percepción, evaluación y expresión de las emociones (Bravo y Urquizo, 2020). El pensamiento divergente plantea la creatividad desde una perspectiva holística para llegar a planteamientos que analizan las partes de un conflicto en un ambiente lúdico, de gran locuacidad y abierto a propuestas irreflexivas, aún a riesgo de ser calificado como un método extravagante. El brainstorming (tormenta de ideas) constituye una herramienta fundamental en la construcción del método de pensamiento lateral, un proceso de activación de la creatividad ya presente en buena parte de las creaciones más experimentales de las vanguardias históricas y en los procesos comunicativos que rigen las relaciones socioculturales de las nuevas generaciones.

\subsection{La creatividad vernácula en la generación centennial y millennial}

La creatividad vernácula se refiere a la acción creativa que interviene en la gestión cotidiana de los contenidos por parte de los usuarios (Barton y Lee, 2012). Burgess (2006) emplea el término vernáculo en referencia a un lenguaje nativo que surge en un contexto social frente al idioma oficial, respondiendo a un modelo de subcultura. Desde una perspectiva antropológica, la cultura vernácula surge como resultado de los conocimientos adquiridos espontáneamente desde las civilizaciones más ancestrales, transmitidas a través de las generaciones mediante la herencia familiar (Cuán, 2010). Generalmente, el término hace referencia a la artesanía y los oficios cuya sabiduría se gesta sobre planteamientos de observación, aprendizaje e imitación para su posterior mejora o reinterpretación (Davila y Macchi, 2018). La cultura vernácula se desarrolla en la esfera informal, cotidiana y local más creativa e innovadora, ajena a los planteamientos comerciales o institucionales (Guzmán-Simón, 2014).

Lejos de constituir un legado extinto o caduco, en la era digital las creaciones vernáculas quedan fijadas en el soporte con mayor alcance y permeabilidad jamás conocido por la humanidad: la World Wide Web. La cultura popular digital se construye individualmente anteponiendo la imagen a la realidad, la producción a la información, los símbolos a los conceptos (McLauglin, 2007). Los millennials, y especialmente la generación zeta, experimentan de forma innata con recursos digitales semióticamente poderosos, incorporándolos a sus discursos digitales en un ejercicio creativo en el que interviene decisivamente el apropiacionismo (Martínez, 2018). 
Según un reciente estudio realizado por Nielsen Global Media (2017), en la actualidad los rasgos definitorios de los jóvenes son: la dimensión tecnológica, la hiperconectividad, la incorporación integral del smartphone a sus tareas diarias, la multipantalla, el uso de las redes sociales, el visionado de contenidos en streaming y la utilización de apps especializadas. En lo relativo a la construcción de nuevos discursos, los millennials y centennials son creadores natos de contenido eminentemente visual, recurren a un uso habitual de la propia imagen, difunden información de índole personal, están concienciados con el medioambiente y les preocupa la hipersocialización. El apropiacionismo forma parte inherente de sus códigos de comunicación, tanto en términos creativos como de consumo en la construcción colectiva de conocimiento mediante una socialización inclusiva a través de las nuevas tecnologías (Sánchez Mateos, 2018). La ironía como recurso retórico y el interés por el feminismo y las culturas urbanas son otras de las claves generacionales (Castro, 2019) que nos consienten comprender el horizonte de expectativas y las coordenadas socio-comunicativas de nuestros estudiantes universitarios más jóvenes para poder integrarlas en nuestra propuesta educativa.

\section{El mash-up como herramienta creativa para la innovación docente}

Entre los principios pedagógicos y comunicativos básicos de la educomunicación, Aparici (2014) destaca la participación, la comunicación dialógica y la autogestión. Estos pilares sirven de modelo para nuestra propuesta de incorporación del apropiacionismo para la construcción de un modelo educativo de carácter innovador, aperturista y transformador. La inclusión de la tecnología supone una gran oportunidad para involucrar a las nuevas generaciones en el aprendizaje a través del desarrollo del pensamiento creativo y lateral. Desde esta investigación apostamos por la introducción del mash-up en el contexto formativo como nuestra estrategia para integrar la fórmula apropiacionista por excelencia de la cultura referencial en la era digital a un nuevo horizonte educativo, alineado con los avances socioculturales y tecnológicos.

Los ejercicios didácticos basados en el apropiacionismo pretenden incorporar la sensibilidad estética al contexto de la educación promoviendo la creatividad y el juego para establecer un escenario innovador en el que convivan enseñanza y práctica (Read y Mantovani, 1999). Asimismo, brindan mecanismos de crecimiento personal que permiten a los estudiantes incorporar a su expertise los conocimientos, habilidades y valores de su contexto sociocultural (Morales, Aguilar y Rodríguez, 2018). Además, este tipo de prácticas invita a reflexionar sobre el concepto de imagen promoviendo nuevas lecturas a través de las imágenes apropiadas y enriqueciendo los discursos artísticos (Esgueva y Nicolau, 2019). También fomentan la actitud crítica propia de los creativos que a lo largo del siglo XX reinterpretaron el arte desde la contracultura con el fin de ofrecer una mirada actualizada de nuestro presente (García Alarcón, 2015). 
Respecto al Grado de Publicidad y Relaciones Públicas, entre los objetivos fundamentales de la titulación figura la "formación en el conocimiento y uso de las tecnologías de la comunicación en los distintos entornos multimedia e hipermedia y (...) el desarrollo de nuevos soportes" (ANECA, 2005, p. 321). Sin embargo, no se señala la importancia de la competencia creativa, pese a que entre los cuatro perfiles profesionales básicos se incluye al "Creativo/a y diseñador/a". Según una encuesta realizada por la Agencia Nacional de Evaluación de la Calidad y Acreditación al alumnado, profesorado y los gestores universitarios de dicho grado, el conocimiento de los métodos del pensamiento creador y su aplicación en el proceso de comunicación publicitaria ocupa la última posición en importancia (ANECA, 2005, p. 276). En cuestión de competencias profesionales, la capacidad y habilidad para dar forma creativa al mensaje se sitúa en la penúltima posición frente al primer lugar ocupado por el dominio tecnológico. No obstante, en el informe final sí se incluye la "capacidad de desarrollo de nuevos enfoques creativos en las tareas asignadas" como una exigencia de formación conforme a la experiencia española y europea y a las tendencias del sector (ANECA, 2005, p. 328). Esto nos lleva a la necesidad de diseñar un método de innovación docente que aúne el desarrollo de la creatividad al manejo de las nuevas tecnologías para la formación de profesionales competitivos y capaces de cumplir con los requerimientos de sus futuros clientes (Roca, 2007).

\subsection{Diseño del método: design thinking y found footage}

El método que proponemos surge al integrar los principios de pensamiento lateral del designthinking y el legado de las prácticas audiovisuales basadas en el found footage a las dinámicas de creatividad vernácula que las nuevas generaciones aplican de forma inconsciente en la gestión personal de sus contenidos en la web.

Recordemos que el design thinking es un método para el desarrollo de la competencia creativa concebido en la década de los años ochenta desde Silicon Valley para detectar retos y proponer soluciones de carácter innovador. Sostenemos la idoneidad de la incorporación de este sistema como herramienta educativa para el desarrollo de las capacidades creativas de los estudiantes a lo largo de su proceso formativo, de modo que puedan enfrentarse a los complejos desafíos del mundo publicitario (Steinbeck, 2011). Para su puesta en práctica, se requiere de una implicación personal basada en la empatía, el pensamiento integrador, el optimismo, el experimentalismo y la colaboración (Brown, 2008). Resulta especialmente relevante para el ámbito académico al fomentar el trabajo en equipos multidisciplinares desde un contexto de lógica disruptiva, impulsando así una libertad creativa que consiente la puesta en marcha de un modelo de acción diferenciador, original y sorprendente.

Por otro lado, el found footage es una técnica idónea para la puesta en práctica del apropiacionismo al caracterizarse por recurrir a materiales audiovisuales preexistentes para generar nuevos modos de representación. Se trata de un procedimiento presente desde los orígenes del cine, que adquirió mayor protagonismo a partir de la década de 
los años setenta y se consolidó en los ochenta como recurso metafílmico. Consiste en la producción de películas formadas mayoritariamente por fragmentos de metraje ajeno que aparecen dispuestos de forma distinta a la versión original, generando nuevos significados. En este procedimiento identificamos dos momentos fundamentales del apropiacionismo: "por un lado, el momento en el que extraemos un elemento de su contexto inicial y le damos una entidad ( $y$, por lo tanto, una significación) autónoma y, por otro lado, el instante en el que decidimos incluirlo en un nuevo contexto alejado del original, resignificándolo" (Serrano, 2013, p. 16).

Mediante nuestra propuesta metodológica pretendemos formular una actualización del found footage a partir del proceso de democratización que consiente el uso de Internet, pues en la actualidad el usuario se convierte en agente activo en el proceso de creación, producción y distribución de los contenidos (UGC) (Fernández-Castrillo, 2014). Hemos adoptado el mash-up como herramienta principal al consentir la combinación de información disponible en la web desde una lógica aditiva o acumulativa basada en procesos interactivos y recombinatorios (Sonvilla-Weiss, 2010). Como pieza audiovisual, "el mash-up responde a una acción de montaje que permite dotar de un sentido nuevo a las piezas preexistentes" (Morales, 2009, p. 136). Los profesionales de la publicidad tienen bien presente que la originalidad de sus propuestas reside en la confluencia de formacontenido, por lo que un relato no es solo lo que se cuenta (historia), sino también cómo se cuenta (discurso) (McKee, 2009; García García, 2004).

\subsection{Lateral visual thinking: una propuesta metodológica para la enseñanza publicitaria}

Introducimos el lateral visual thinking como metodología de innovación docente para los estudios de Publicidad. Se trata de un método disruptivo de creación audiovisual basado en la apropiación de obras preexistentes en entornos conectivos, sometidas a un proceso de reinterpretación, edición y postproducción con el objetivo de elaborar una pieza creativa de carácter innovador. Recuperamos las pautas del discurso apropiacionista de Agudelo (2019) (recepción, interpretación, apropiación) y sumamos la práctica del razonamiento abstracto y el pensamiento divergente para el desarrollo de la libre asociación de ideas. A continuación, expondremos su puesta en práctica a través de una actividad estructurada en tres fases con sus correspondientes tareas:

1. Observación. Como hemos podido comprobar, los millennials y centennials actúan de forma hipersocializada como comunidades digitales (Álvaro, 2015). Por ello, se propone una organización del trabajo en equipos multidisciplinares basada en la teoría de roles de Belbin (2010).

a) Archivo profundo. Para garantizar la implicación de todos los integrantes y el máximo rendimiento del grupo a través de la empatía, arrancaremos con una dinámica participativa 
en la que cada estudiante resumirá su trayectoria vital señalando sus inspiraciones, referencias e intereses, incluyendo también sus expectativas profesionales. Se prestará especial atención a su huella digital como usuarios gamers y su influencia en las redes sociales.

b) Briefing. Nos referimos al objeto del ejercicio como briefing empleando el término profesional con el que se hace referencia al encargo del cliente al anunciante (Roca, 2007). El docente indicará el tema de la pieza final, pudiendo optar por una cuestión específica o un concepto abierto. En ambos casos, permitirá a los estudiantes absoluta libertad en el proceso creativo, limitándose a dinamizar el proceso creativo y la interacción en clase (De la Torre y Violant, 2002).

c) Brainstorming. La tormenta de ideas es una de las claves del pensamiento lateral para la estimulación del asociacionismo: los miembros del grupo expondrán todas las ideas, interpretaciones y asociaciones que les sugiere el tema a tratar, sin filtro alguno (González Rivero, 2008). De este modo, emergerá la yuxtaposición de conceptos aparentemente dispares desde un ambiente lúdico y distendido.

d) Observación encubierta activa. La observación encubierta consiste en presenciar a un grupo de gente sin que se dé cuenta, permitiendo que se exprese sin intervenir ni tratar de influir sobre sus opiniones (Provenza y Michel, 2017). En el caso que nos ocupa, nos referimos a una observación activa ya que todos los sujetos participan exponiendo sus ideas. Los observadores escuchan empáticamente, sin descartar ideas, emitir juicios de valor o poner en duda la viabilidad de las sugerencias (Saiz, 2005).

2. Interpretación. Esta segunda fase consiste en el análisis de las ideas planteadas en la fase inicial para identificar los símbolos, códigos y asociaciones que conduzcan a la formulación concreta del mash-up desde el establecimiento de una línea de trabajo y unos objetivos precisos.

e) Análisis del brainstorming. Tiene dos objetivos: identificar los conflictos y oportunidades; y descartar las ideas desprovistas de símbolos y significado. Se potencia la generación de ideas por asociación y el ejercicio de síntesis para simplificar las propuestas planteadas (Selva-Ruiz y Domínguez-Liñán, 2018).

f) Visionado. Se trata de una fase de documentación que consiste en localizar el material disponible, directa o indirectamente relacionado con las ideas seleccionadas y el enfoque narrativo escogido. Denominamos este ejercicio como "visionado" empleando el término referido al proceso de búsqueda de material de archivo propio del cine documental cuya finalidad es dotar de veracidad al contenido (Mamblona, 2012). Esta tarea puede realizarse en equipo o de forma individual, adjudicando un reparto de temáticas a cada integrante. Se adoptará la plataforma YouTube como fuente principal de contenidos generados por usuarios gracias a su accesibilidad, su vasto catálogo de contenido audiovisual en constante actualización y la efectividad de su buscador (Ramírez-Ochoa, 2016). 
g) Selección. La recopilación de las piezas de YouTube que participarán en el corte final resulta decisiva al determinar el punto de vista narrativo y la nueva intencionalidad del material respecto a su versión original. No existen límites en relación con el criterio de selección temporal y de formato: videoclips, telediarios, documentales, programas, anuncios, fotografías, pinturas, videojuegos, etc. de cualquier época (McGranahan, 2010). Para facilitar la ordenación del material, se recurrirá al diagrama de prioridades que permite establecer un criterio jerárquico de los objetivos, funciones y restricciones (González, Villarroel y Viveros, 2017). Cabe destacar que, al tratarse de ejercicios educativos, cuya finalidad es el aprendizaje y no la difusión, no existen restricciones legales en el uso de imágenes y música.

h) Moodboard. El grupo representará los clips seleccionados en un collage visual formado por capturas de pantalla identificativas de cada pieza. Esto permitirá tener un mapa general de los videos seleccionados y una imagen mental del estilo formal (Vallet, 2005).

3. Apropiación. Siguiendo el curso natural de la cultura apropiacionista, esta fase culmina la elaboración del mash-up como creación original.

i) Narrativa. El objetivo de este punto será establecer una ordenación del material en bruto (fragmentos de videoclips descargados) para construir una narrativa o storytelling del producto final que dé lugar a una "unidad sinfónica” (Mckee, 2009, p. 49).

j) Construcción simbólica. Los clips resultan fácilmente identificables al integrar fragmentos icónicos de la actual cultura popular. Se erigen, por tanto, como metáforas del imaginario colectivo en la era digital mediante la resignificación de símbolos ampliamente reconocibles. En esta fase los estudiantes llevarán a cabo una doble versión digital del "viaje del héroe" (Campbell, 2015), como creadores del propio argumento del mash-up y enfrentándose al reto narrativo de generar un hilo conductor a partir del material apropiado y posteriormente transformado.

k) Edición. Cada grupo se enfrenta en esta fase a la construcción definitiva del mash-up a través del montaje de los clips (entre 1,5 y 2 minutos de duración). Para ello recomendamos el uso de programas similares a DaVinci, al ser gratuito, intuitivo y con herramientas profesionales de montaje y etalonaje accesibles para todo tipo de perfiles. Debido a las particularidades del género, sugerimos la técnica del montaje sobre música que establece la duración del plano por las variaciones rítmicas, similar al de las películas sinfónicas (Sánchez López y García Gómez, 2009).

l) Postproducción. La banda sonora será el componente clave para marcar el ritmo de la pieza final. Además, se pueden añadir efectos que enfaticen la intencionalidad deseada a través de las transiciones, el color, flashbacks o flash forwards, glitch, sobreimpresiones, títulos, pantalla dividida, voz en off, silencios, etc. El docente deberá recordar a los estudiantes que dichos recursos deben gestionarse siempre a favor de la intención dramática y no como meros ornamentos. 
m) Validación. Cada grupo mostrará su mash-up al resto de equipos para testear la comprensión del mensaje y el impacto del discurso formal antes de la presentación final. Se trata de un proceso de aprendizaje para ambas partes, basado en la interacción constructiva a partir de la comunicación, el respeto y la crítica positiva (Castañeda, 2014). Esta tarea reproduce el llamado customer journey (viaje del cliente), es decir, la experiencia percibida por el usuario final.

n) Presentación final. Cada grupo presentará su mash-up siguiendo la técnica del elevator pitch, una de las tendencias en auge en publicidad y comunicación que consiste en una breve introducción (entre 45 segundos y 2 minutos) en la que se recrea un trayecto de ascensor en el que se intenta persuadir a un potencial cliente de forma asertiva y sintética (Ríos, 2018).

El lateral visual thinking es, en definitiva, un método educativo para el desarrollo de la competencia creativa y el pensamiento divergente en entornos conectivos a través del apropiacionismo digital. En la elaboración de la propuesta se ha tenido en cuenta la condición natural de creadores de contenidos de los estudiantes-produsuarios para intentar hacer más atractiva la enseñanza de la creatividad e innovación en comunicación, especialmente publicitaria.

\section{Conclusiones}

En el presente, guiados por procesos de creatividad vernácula, nuestros estudiantes más jóvenes ejercitan diariamente el apropiacionismo fuera de los circuitos académicos, mediante el intercambio de múltiples recursos, propios y ajenos, a través de las redes sociales, poniendo en práctica el pensamiento colectivo y la acción social. Es, por tanto, necesario incorporar a la enseñanza los procesos consustanciales a las dinámicas de interrelación social, adquisición de conocimiento e intercambio de información en las redes sociodigitales. En el actual ecosistema digital se pueden observar hibridaciones, resignificaciones e intertextualidades que dan una idea concreta de la evolución de los mecanismos de producción, distribución y consumo de contenidos. La formación de profesionales competentes en Publicidad requiere de una metodología estructurada en materia de creatividad que permita implicar a los estudiantes en su formación estableciendo un punto de encuentro entre sus hábitos de creatividad vernácula y los planes de estudio existentes. Así pues, es necesario establecer puntos de conexión entre las aptitudes digitales de los millennials y centennials como creadores intuitivos de contenido y acciones educativas que permitan a los docentes enfatizar las habilidades creativas imprescindibles para el desempeño futuro de la actividad profesional publicitaria, así como promover una implicación activa, participativa e interactiva por parte de los estudiantes. 
Desde esta investigación se ha pretendido señalar la importancia del mash-up como herramienta educativa para dar forma creativa al mensaje publicitario en la era digital a partir de una nueva interpretación de la realidad que diera origen a un discurso de autoría propia partiendo de la documentación y selección de materiales preexistentes de libre acceso desde la World Wide Web. A través de la construcción de nuevos modelos discursivos en entornos conectivos, se ha pretendido fomentar el trabajo en equipo, el espíritu multitarea y el desarrollo de algunas de las destrezas y habilidades básicas para la formación de los futuros publicistas, como pueden ser la capacidad para evolucionar hacia lo desconocido mediante la perspicacia y el ingenio con el fin de hallar soluciones eficaces a problemas inéditos desde la creatividad. Una contribución esencial, para hacer frente a la amenaza de la creatividad programadora que ya ocupa las principales líneas de I+D de empresas como Microsoft. Por tanto, sostenemos que una formación dirigida al desarrollo del pensamiento lateral y creativo contribuirá en gran medida a la cualificación de los profesionales publicitarios del siglo XXI.

\section{Agradecimientos}

La presente investigación forma parte del proyecto “Digital Media Culture: Intercreativity and Public Engagement" (Venice Centre for Digital and Public Humanities, Università Ca' Foscari Venezia), financiado por el Ministerio Italiano de Educación, Universidades e Investigación, MIUR. También se integra en la línea investigación "Arqueología de medios digitales: intermedialidad, narrativas transmedia y UGC" del grupo Televisión-cine: memoria, representación e industria (TECMERIN, Universidad Carlos III de Madrid).

\section{Referencias}

Agudelo Vergara, A. C. (2019). La apropiación en la comunicación y en la educación. Construcción teórica de la apropiación desde las experiencias comunicativas de estudiantes universitarios. Signo y Pensamiento, 38(75). doi: https://doi.org/f4wp

Álvaro Martín, A. (2015). Jóvenes y generación 2020. Revista de estudios de la juventud, 108, 9-20. Recuperado de https://cutt.ly/kjqJOgN

ANECA (2005). Libro Blanco. Títulos de grado en Comunicación. Recuperado de https://cutt.ly/HjqJ7pg Aparici, R. (coord.) (2014). Conectados en el ciberespacio. Madrid: UNED.

Ballesteros Guerra, J. C., Calderón Gómez, D., Kuric Kardelis, S., Megías Quirós, I. y Sanmartín Ortí, A. (2020). Barómetro Jóvenes y expectativa tecnológica 2020. Madrid: Centro Reina Sofía sobre Adolescencia y Juventud. Recuperado de https://cutt.ly/PjqZj1Y

Barton, D. y Lee, C. K. M. (2012). Redefining Vernacular Literacies in the Age of Web 2.0. Applied Linguistics, 33(3). doi: https://doi.org/f35c8f

Belbin, M. R. (2010). Management teams: why they succeed or fail. Amsterdam; Oxford: ButterworthHeinemann.

Bravo Mancero, P. y Urquizo Alcivar, A. M. (2020). Razonamiento lógico abstracto e inteligencia emocional: trayectorias en la formación de estudiantes universitarios. Sophia, Colección de Filosofía de la Educación, 21, 179-208. doi: 10.17163/soph.n21.2016.08

Brown, T. (2008). Design thinking. Harvard Business Review, Sept., 3-10. 
Bruns, A. (2008). Blogs, Wikipedia, Second Life, and beyond: from production to produsage. New York: Peter Lang.

Burgess, J. (2006). Hearing Ordinary Voices: Cultural Studies, Vernacular Creativity and Digital Storytelling. Continuum, 20(2), 201-214. doi: https://doi.org/cmjnf4

Campbell, J. (2015). El poder del mito. Madrid: Capitán Swing.

Castañeda Millán, F. J. (2014). Hacia una interacción constructiva: una propuesta de alumnos de preparatoria. RIDE, Revista Iberoamericana para la Investigación y el Desarrollo Educativo, 5(9), 42-55. Recuperado de https://cutt.ly/DjqLWpu

Castro Córdoba, E. (2019). El trap: filosofía millennial para la crisis en España. Madrid: Errata Naturae.

Cuán Aguilar, M. G. (2010). Arquitectura vernácula. Ciencias, 98, 10-12. Recuperado de https://cut t.ly/GjqLHml

Davila Cordido, M. y Macchi Jánica, C. (2018). La conservación de los valores cromáticos de la arquitectura vernácula: casos de las rancherías Wayúu. Geconservación, 14, 52-63. Recuperado de https://cutt.ly/5jqLK7m

De Bono, E., y Gombau Arnau, A. (2008). El pensamiento lateral práctico. Barcelona: Paidós Ibérica.

De Bono, E. (1989). Pensamiento lateral. Manual de creatividad. Barcelona: Paidós Ibérica.

De la Torre, S. y Violant, V. (2002). Estrategias creativas en la enseñanza universitaria. Creatividad y sociedad, 3, 21-47. Recuperado de https://cutt.ly/mkRTVJE

Domínguez Alfonso, R. (2011). Reconsiderando el papel de los docentes ante la sociedad de la información.Etic@net, 11, 179-195. Recuperado de https://cutt.ly/Ocntdkn

Esgueva López, V. y Nicolau López, N. (2019). Apropiacionismo en el arte, la legitimidad de la imagen sustraída. En IV Congreso Internacional de Investigación en Artes Visuales ANIAV 2019 Imagen [N] Visible, Universitat Politècnica de València, 226-231. doi: https://doi.org/f4wk

Fernández-Castrillo, C. (2014). Prácticas transmedia en la era del prosumidor: Hacia una definición del Contenido Generado por el Usuario. CIC. Cuadernos de Información y Comunicación, 19, 53-67. doi: https://doi.org/f4wj

Foro Económico Mundial. (2020). Schools of the Future: Defining New Models of Education for the Fourth Industrial Revolution. Ginebra: World Economic Forum. Recuperado de https://cutt.ly/CjqL4iO

García Alarcón, A. (2015). Lazos, apropiaciones y transgresiones de estrategias de la iconosfera publicitaria en las artes visuales. Una perspectiva crítica desde el arte español actual. Imafronte, 24, 279-300. Recuperado de https://cutt.ly/ vjqZwY5

García García, F. (2004). Posibilidades creativas de la imagen. Inteligencia y creatividad. Icono 14. Revista de comunicación y nuevas tecnologías, 2. Recuperado de https://cutt.ly/pjqZyat

González Rivero, M. C. (2008). Tormenta de ideas: iqué tontería tan genial! ACIMED, 17(4). Recuperado de https://cutt.ly/MkRYkD2

González, J., Villarroel, M. y Viveros, F. (2017). Función de Priorización para tomar decisiones a partir de diagnósticos cuantitativos: Propuesta. Ciencia, Docencia y Tecnología, 28(55), 180-194. Recuperado de https://cutt.ly/zkRYv89

Gray, A. (2020). The 10 skills you need to thrive in the Fourth Industrial Revolution. World Economic Forum. Recuperado de https://cutt.ly/WjqZI9p

Guzmán-Simón, F. (2014). Prácticas vernáculas de lectura y escritura. Diccionario Digital de nuevas formas de lectura y escritura. Recuperado de https://cutt.ly/GjqZvj7 
Luengo Navas, J. J., Luzón Trujillo, A. y Torres Sánchez, M. (2008). Las reformas educativas basadas en el enfoque por competencias: una visión comparada. Profesorado. Revista de currículum y formación del profesorado, 12(3). Recuperado de https://cutt.ly/pkRloZ4

Mamblona Agüera, R. (2012). Las nuevas subjetividades en el cine documental contemporáneo. Análisis de los factores influyentes en la expansión del cine de lo real en la era digital [tesis doctoral]. Barcelona: Universitat Oberta de Catalunya. Recuperado de https://cutt.ly/rjqCmZt

Martín-Barbero, J. (1987). De los medios a las mediaciones. Barcelona: Gustavo Gili.

Martínez, N. (2018). La generación zeta es autodidacta, sensible con el medio ambiente y adicta a la tecnología. Barcelona: Universitat Oberta de Catalunya. Recuperado de https://cutt.ly/gjqCG0g

McGranahan, L. (2010). Bastards and Booties: Production, Copyright, and the Mashup Community. Trans, Revista cultural de música, 14. Recuperado de https://cutt.ly/ejqC6Vo

McKee, R. (2009). El Guion Story: sustancia, estructura, estilo y principios de la escritura de guiones. Barcelona: Alba.

McLauglin, T. (2007). Beyond the Reflective Teacher. Educational Philosophy and Theory, 31(1). doi: https://doi.org/f42p

Morales Páez, Y., Aguilar Hernández, V. y Rodríguez Delgado, C. (2018). Los medios de enseñanza para la apropiación de contenidos profesionales. Mendive, 16(1). doi: https://doi.org/f42p

Morales Morante, L. F. (2009). Montaje o edición: un diseño y modelo de clasificación basado en objetivos de comunicación. Anagramas, Rumbos y Sentidos de la Comunicación, 7(14), 133-141. doi: 10.22395/angr.v7n14a9

Nielsen Global Media (2017). Millennials on millennials. Digital music and communication. Recuperado de https://cutt.ly/pjqZWKu

Perrenoud, P. H. (2000). L'approche par competences ¿Une réponse à l' échec scolaire? AQPC Réussir au collégial. Actes du Colloque de l'association de pédagogie collégiale, Montréal, Sept. Recuperado de https://cutt.ly/BjqVbzT I

Porto, M. (2008). Evaluación para la competencia creativa en la educación universitaria. Cuadernos de la Facultad de Humanidades y Ciencias Sociales - Universidad Nacional de Jujuy, 35, 77-90. Recuperado de https://cutt.ly/wjqVENm

Prado, L. E., Viteri, M. J. y Rojas, M. P. (2017). Aporte del pensamiento lateral al desarrollo de la inteligencia lingüística. Estudios culturales y sociales, vol. 4, 13(2), 269-281. Recuperado de htt ps://cutt.ly/kjqVOKv

Provenza, A. y Michel Fariña, J. J. (2017). Covert Research: El debate ético sobre la investigación encubierta en las ciencias sociales. Aesthethika, Revista Internacional de estudio e investigación interdisciplinaria sobre subjetividad, política y arte, 13(2), 35-49. Recuperado de https://cutt.ly/ ZjqVFW3

Ramírez-Ochoa, M. I. (2016). Posibilidades del uso educativo de YouTube. Ra Ximhai, 12(6), 537-546. Recuperado de https://cutt.ly/ojqVXII

Read, H. y Mantovani, J. (1999). Educación por el Arte. Barcelona: Paidós.

Ríos Cardona, M. P. (2018). Elevator pitch, herramienta estratégica para proyectos de seguridad y paz en el posconflicto. Revista Científica General José María Córdova, 16(21), 35-59. doi: https:/ /doi.org/f42q

Roca, D. (2007). El briefing creativo en las agencias de publicidad: una aproximación cualitativa al caso español. Zer, Revista de Estudios de Comunicación, 12(23), 165-182. Recuperado de https ://cutt.ly/BkRPDVb 
Saiz Segarra, M. A. (2005). Cómo potenciar la generación de nuevas ideas en el Proceso de Innovación Tecnológica [tesis doctoral]. Barcelona: Universitat Politècnica de Catalunya. Recuperado de $\underline{\mathrm{h}}$ ttps://cutt.ly/PjqMSj5

Sánchez López, J. A. y García Gómez, F. (coords.) (2009). Historia, estética e iconografía del videoclip musical. Málaga: Universidad de Málaga.

Sánchez Mateos, A. (16 de julio de 2018). 10 datos sobre la Generación Zeta. La Vanguardia. Recuperado de https://cutt.ly/EjqML9P

Selva-Ruiz, D. y Domínguez-Liñán, R. (2018). Las técnicas de generación de ideas: revisión y análisis de su uso en las agencias publicitarias españolas. Área Abierta, Revista de comunicación audiovisual y publicitaria, 18(3), 371-387. doi: https://doi.org/f42r

Serrano Vidal, A. (2013). Apropiacionismo, remezcla y postproducción: el Found Footage en el siglo XXI. En Crespo Fajardo, J. L. (coord.), Estéticas del Media Art. Málaga: Eumed.net, 15-36.

Sonvilla-Weiss, S. (2010). Introduction: Mashups, Remix practices and the Recombination of Existing Digital Content. En Sonvilla-Weiss, S. (ed.), Mashup Cultures. Viena: Springer-Verlag, 8-23.

Steinbeck, R. (2011). El "design thinking" como estrategia de creatividad en la distancia. Comunicar, 19(37), 27-35. doi: https://doi.org/fn55jw

Trillo, F. (2005). Competencias Docentes y Evaluación Auténtica: ¿falla el protagonista? Revista Perspectiva Educacional, 45, 86-102. Recuperado de https://cutt.ly/Zjq0ABW

UNESCO. (2018). Re | pensar las políticas culturales. Creatividad para el Desarrollo. París: Organización de las Naciones Unidas para la Educación, la Ciencia y la Cultura. Recuperado de https://cut t.ly/njq0Be7

Vallet, G. (2005). eBranding. La creación de marca digital en la era de la conectividad [tesis doctoral]. Barcelona: Universidad Autónoma de Barcelona. Recuperado de https://cutt.ly/hjq02Hj

Villarroel, J. (2011). ¿Qué es el pensamiento lateral? Revista Pensamiento lateral, 1, 4. Ibarra, Ecuador. Recuperado de https://cutt.ly/Qjq2wSi 